\title{
Fixed-point theorems for mappings satisfying the ordered contractive condition on noncommutative spaces
}

\author{
Qiaoling Xin and Lining Jiang*
}

"Correspondence:

jianglining@bit.edu.cn

School of Mathematics and

Statistics, Beijing Institute of

Technology, Beijing, 100081, China

\begin{abstract}
In the paper, we introduce noncommutative Banach spaces which generalize the concept of Banach spaces, and the k-ordered contractive condition; we then discuss an ordered structure and several properties on noncommutative Banach spaces. Moreover, some fixed-point theorems for mappings with the k-ordered contractive condition on noncommutative Banach spaces are presented. In addition, we investigate the existence and uniqueness of fixed points for an integral equation of Fredholm type.
\end{abstract}

MSC: $47 \mathrm{H} 10$

Keywords: noncommutative Banach space; ordered contractive condition; fixed-point theorem

\section{Introduction}

The well-known fixed-point theorem of Banach [1] is a very important tool for solving existence problems in many branches of mathematics and physics. There are a large number of generalizations of the Banach contraction principle in the literature (see [2-14] and others). The theorem has been generalized in two directions. On the one side, the usual contractive condition is replaced by weakly contractive conditions. On the other side, the action spaces are replaced by metric spaces endowed with an ordered or partially ordered structure. In particular, there is much interest in obtaining the existence and uniqueness of fixed points for self-maps by altering the action spaces. In this direction, Dhage et al. [8] addressed a new category of fixed-point problems for a self-map with the help of ordered Banach spaces. Further improvements in those spaces were found in [14]. In recent years, Ran and Reurings [13], O'Regan and Petruşel [12] and others started the investigations concerning a fixed-point theory in ordered metric spaces. Later, many authors followed this concept by introducing and investigating the different types of contractive mappings, e.g., in [6] Caballero et al. considered contractive-like mappings in ordered metric spaces and applied their results in ordinary differential equations. Some interesting fixed-point theorems concerning partially ordered metric spaces can also be found in $[3,5]$.

The results obtained by Huang and Zhang [9] have become of interest for many scholars. They reconsidered the Banach contraction principle by initiating a new concept of cone metric spaces. Recently, also, the existence of fixed points for the given contractive type mappings in partially ordered cone metric spaces was investigated (see $[4,10])$.

○2014 Xin and Jiang: licensee Springer. This is an Open Access article distributed under the terms of the Creative Commons Attribution License (http://creativecommons.org/licenses/by/2.0), which permits unrestricted use, distribution, and reproduction in any medium, provided the original work is properly cited. 
The purpose of this paper is to present some fixed-point theorems for mappings satisfying the ordered contractive condition in the context of noncommutative metric spaces which are noncommutative sense of those in [9].

The paper is organized as follows: we firstly introduce a noncommutative Banach space and the $k$-ordered contractive condition, and then discuss the ordered structure and several properties on noncommutative Banach spaces. Moreover, some fixed-point theorems on this space are established. Finally, we investigate the existence and uniqueness of fixed points for integral equation of Fredholm type.

Throughout this paper, the letters $\mathbb{R}, \mathbb{R}^{+}, \mathbb{N}$ will denote the sets of all real numbers, nonnegative real numbers and natural numbers, respectively.

To begin with, we introduce some definitions and properties which will be used later.

Definition 1.1 Let $E$ be a group with a unit $e$ and suppose that there exists a metric $d$ on $E$ such that $(E, d)$ is a complete metric space. $E$ is said to be a noncommutative Banach space if the following conditions hold:

(1) for any $x, y, z \in E$, we have $d(x z, y z)=d(x, y)$;

(2) there exists a binary continuous operation

$$
F: \mathbb{R} \times E \rightarrow E, \quad(\alpha, x) \mapsto x^{\alpha}
$$

such that $F(-1, x)=x^{-1}$ is exactly the inverse of $x$ in the group $E$ and $F(0, x)=x^{0}=e$ is the unit in the group $E$, and that

$$
F(m n, x)=F(m, F(n, x)), \quad F(m+n, x)=F(m, x) F(n, x)
$$

for $m, n \in \mathbb{R}, x \in E$;

(3) for any $x \in E$, there exists a constant $M_{x}>0$ such that

$$
d\left(x^{\alpha}, e\right) \leq M_{x}|\alpha|, \quad \forall \alpha \in \mathbb{R} .
$$

In particular, if there exists a constant $M>0$ such that $d\left(x^{\alpha}, e\right) \leq M|\alpha|$ for $x \in E, \alpha \in \mathbb{R}$, then $E$ is said to be uniformly bounded.

Let $E$ be a uniformly bounded noncommutative Banach space. Taking $\alpha=1$, we conclude that $d(x, e) \leq M$, which together with the triangular inequality yields $d(x, y) \leq 2 M$. This shows $E$ is bounded.

Example 1.1 All Banach spaces are noncommutative Banach spaces. Let $(X,\|\cdot\|)$ be a Banach space, then $(X,+)$ is a group with a unit $\theta$, and there exists a metric $d$ induced by the norm $\|\cdot\|$ such that $(X, d)$ is a complete metric space. Firstly, the metric $d$ satisfies $d(x+z, y+z)=d(x, y)$ for $x, y, z \in X$. Secondly, there exists a binary continuous mapping $F: \mathbb{R} \times X \rightarrow X,(k, x) \mapsto k x$ satisfying the condition (2) in Definition 1.1. Finally, for any $x \in X$, there exists a constant $M_{x}=\|x\|+1>0$ such that $d(k x, \theta)=\|k x\|=|k|\|x\| \leq|k| M_{x}$, $\forall k \in \mathbb{R}$. According to the definition, $X$ is a noncommutative Banach space. 
Example 1.2 Let $\mathbb{R}^{n}$ be the standard $n$-dimensional vector space over $\mathbb{R}$. Define

$$
d(x, y)=\sum_{i=1}^{n} \frac{1}{2^{i}} \frac{\left|x_{i}-y_{i}\right|}{1+\left|x_{i}-y_{i}\right|}
$$

for $x=\left(x_{1}, x_{2}, \ldots, x_{n}\right), y=\left(y_{1}, y_{2}, \ldots, y_{n}\right) \in \mathbb{R}^{n}$. Clearly, $\mathbb{R}^{n}$ is a complete metric space. In order to verify that $\mathbb{R}^{n}$ is a noncommutative Banach space. It suffices to show that for any $x=\left(x_{1}, x_{2}, \ldots, x_{n}\right) \in \mathbb{R}^{n}$, there exists a constant $M_{x}>0$ such that $d(k x, \theta) \leq M_{x}|k|$, for $k \in \mathbb{R}$, where $\theta$ is unit in $\mathbb{R}^{n}$. Indeed, choose $M_{x}=\left\{1,\left|x_{1}\right|,\left|x_{2}\right|, \ldots,\left|x_{n}\right|\right\}$, and we get $d(k x, \theta) \leq$ $M_{x}|k|$.

Example 1.3 Suppose that $H$ is a Hilbert space and $U(H)$ is the unitary group of $H$. Put

$$
d(S, T)=\left\|S T^{-1}-I\right\|=\|S-T\| \quad \forall S, T \in U(H),
$$

then $U(H)$ is a complete metric space as a subset of $B(H)$, where $B(H)$ denotes the algebra of all bounded linear operators on $H$. Moreover, for any $T \in U(H)$ and $\alpha \in \mathbb{R}$, set

$$
T^{\alpha}=\int_{0}^{2 \pi} e^{i \alpha \theta} d E_{\theta}
$$

where $E_{\theta}$ means the spectral measure associated with the operator $T$. Notice that

$$
d\left(T^{\alpha}, I\right)=\left\|\int_{0}^{2 \pi}\left(e^{i \alpha \theta}-1\right) d E_{\theta}\right\| \leq \sup _{\theta \in[0,2 \pi]}\left|e^{i \alpha \theta}-1\right| \leq 2 \pi|\alpha|
$$

for $\alpha \in \mathbb{R}$. Then $U(H)$ is a uniformly bounded noncommutative Banach space.

Definition 1.2 Let $E$ be a noncommutative Banach space. $P$ is a subset of $E$ satisfying the following conditions:

(1) $P$ is nonempty, closed, and $P \neq\{e\}$;

(2) $x, y \in P$ and $\alpha, \beta \in \mathbb{R}^{+}$implies $x^{\alpha} y^{\beta} \in P$;

(3) $P \cap P^{-1}=\{e\}$ where $P^{-1}=\left\{x^{-1}: x \in P\right\}$.

Then $P$ is called a cone in $E$.

Given a cone $P$ in a noncommutative Banach space $E$, a relation can be introduced as follows:

$$
x \lesssim y \quad \Longleftrightarrow \quad y^{\beta} x^{-\beta} \in P \quad \text { for all } \beta \in[0,1]
$$

One can show that ' $\lesssim$ ' is a partial ordering in $E$ with respect to $P$. In fact:

(i) For $x \in E, x^{\beta} x^{-\beta}=x^{\beta-\beta}=x^{0}=e \in P$ for all $\beta \in[0,1]$. This implies that $x \lesssim x$.

(ii) If $x \lesssim y$ and $y \lesssim x$, then $y^{\beta} x^{-\beta} \in P$ and $\left(y^{\beta} x^{-\beta}\right)^{-1}=x^{\beta} y^{-\beta} \in P$ for all $\beta \in[0,1]$. By $P \cap P^{-1}=\{e\}$, we get $y^{\beta}=x^{\beta}$, which implies that $y=x$.

(iii) If $x \lesssim y$ and $y \lesssim z$, then $y^{\beta} x^{-\beta} \in P$ and $z^{\beta} y^{-\beta} \in P$ for all $\beta \in[0,1]$, which together with the condition (2) in Definition 1.2 can infer $z^{\beta} x^{-\beta} \in P$. This shows that $x \lesssim z$. 
Definition 1.3 A cone $P \subseteq E$ is called normal if there is a number $N>0$ such that

$$
e \lesssim x \lesssim y \quad \Rightarrow \quad d(x, e) \leq N d(y, e), \quad \forall x, y \in E
$$

The least positive number $N$ satisfying the above is called the normal constant of $P$. It is clear that $N \geq 1$.

Remark 1.1 Let $x \in P, \alpha \in \mathbb{R}$, then the following relation holds:

$$
\left\{\begin{array}{l}
x \lesssim x^{\alpha}, \quad \alpha \geq 1 \\
x^{\alpha} \lesssim x, \quad \alpha<1 .
\end{array}\right.
$$

Indeed, for any $\beta \in[0,1]$, if $\alpha \geq 1$, then $\left(x^{\alpha}\right)^{\beta} x^{-\beta}=x^{(\alpha-1) \beta} \in P$ since $P$ is a cone, thus we can get $x \lesssim x^{\alpha}$; if $\alpha<1$, then $x^{\beta}\left(x^{\alpha}\right)^{-\beta}=x^{(1-\alpha) \beta} \in P$, which means $x^{\alpha} \lesssim x$.

Definition 1.4 For $u, v \in E$, if either $u \lesssim v$ or $v \lesssim u$ holds, we say that $u$ and $v$ are comparable, denoted

$$
\vee(u, v)= \begin{cases}u, & v \lesssim u \\ v, & u \lesssim v\end{cases}
$$

From the above definitions, we have the following properties.

Lemma 1.1 Suppose that $P$ is a cone in E. For $u, v \in E$, we have:

(1) Set $u \lesssim v$, then $u^{\alpha} \lesssim v^{\alpha}$ holds for any $0 \leq \alpha \leq 1$.

(2) If $u$ and $v$ are comparable, then $u v^{-1}$ and $v u^{-1}$ are comparable, and furthermore $e \lesssim \vee\left(u v^{-1}, v u^{-1}\right)$.

(3) If $u$ and $v$ are comparable, then $d\left(\vee\left(u v^{-1}, v u^{-1}\right), e\right)=d(u, v)$.

(4) (compatibility) Let $\left\{u_{n}\right\},\left\{v_{n}\right\} \subset E$, $u_{n}$ and $v_{n}$ be comparable for all $n \in \mathbb{N}$. If $u_{n} \rightarrow u_{0}$, $v_{n} \rightarrow v_{0}$, then $u_{0}$ and $v_{0}$ are comparable.

Proof (1) Let $u \lesssim v$, we have $v^{\beta} u^{-\beta} \in P$ for all $\beta \in[0,1]$. Since $\alpha \beta \in[0,1]$ for any $\alpha \in[0,1]$, we see $v^{\alpha \beta} u^{-\alpha \beta} \in P$, which implies that $u^{\alpha} \lesssim v^{\alpha}$.

(2) Without loss of generality, one can suppose that $u \lesssim v$, which means $v u^{-1} \in P$. Using Remark 1.1, one can see $u v^{-1} \lesssim v u^{-1}$. Furthermore $\left(v u^{-1}\right)^{\beta} e^{-\beta}=\left(v u^{-1}\right)^{\beta} \in P$ for all $\beta \in[0,1]$, which implies $e \lesssim v u^{-1}$, and therefore $e \lesssim \vee\left(u v^{-1}, v u^{-1}\right)$.

(3) Assume that $u \lesssim v$, then $\vee\left(u v^{-1}, v u^{-1}\right)=v u^{-1}$. It follows immediately from Definition 1.1 that

$$
d\left(\vee\left(u v^{-1}, v u^{-1}\right), e\right)=d\left(v u^{-1}, e\right)=d(v, u)=d(u, v) .
$$

(4) Since $u_{n}$ and $v_{n}$ are comparable for all $n$, we can suppose that there exist two subsequences $u_{n_{k}}$ and $v_{n_{k}}$ such that $u_{n_{k}} \lesssim v_{n_{k}}$ for all $k$. Note that for all $\beta \in[0,1]$

$$
\begin{aligned}
d\left(v_{n_{k}}^{\beta} u_{n_{k}}^{-\beta}, v_{0}^{\beta} u_{0}^{-\beta}\right) & \leq d\left(v_{n_{k}}^{\beta} u_{n_{k}}^{-\beta}, v_{0}^{\beta} u_{n_{k}}^{-\beta}\right)+d\left(v_{0}^{\beta} u_{n_{k}}^{-\beta}, v_{0}^{\beta} u_{0}^{-\beta}\right) \\
& =d\left(v_{n_{k}}^{\beta}, v_{0}^{\beta}\right)+d\left(u_{n_{k}}^{-\beta}, u_{0}^{-\beta}\right) .
\end{aligned}
$$


It follows directly that

$$
d\left(v_{n_{k}}^{\beta} u_{n_{k}}^{-\beta}, v_{0}^{\beta} u_{0}^{-\beta}\right) \rightarrow 0
$$

As $P$ is closed, one obtains $v_{0}^{\beta} u_{0}^{-\beta} \in P$. This says that $u_{0} \lesssim v_{0}$.

\section{Fixed-point theorems on noncommutative Banach spaces}

From now on, we always suppose that $E$ is a noncommutative Banach space with a partial ordering $\lesssim$ induced by a normal cone $P$ with the normal constant $N$. And some fixedpoint theorems for mappings on $E$ satisfying the ordered contractive condition will be presented. Let us begin with the following theorem.

Theorem 2.1 Let $A: E \rightarrow E$ be a continuous mapping and suppose that the following two assertions hold.

(1) There exists a constant $k \in(0,1)$ such that for all $u, v \in E$, if $u$ and $v$ are comparable, then $\mathrm{Au}$ and $\mathrm{Av}$ are comparable and furthermore

$$
\vee\left(A v(A u)^{-1}, A u(A v)^{-1}\right) \lesssim \vee\left(v u^{-1}, u v^{-1}\right)^{k} .
$$

In this case, we say $A$ satisfies the $k$-ordered contractive condition.

(2) There exists $x_{0} \in E$ such that $x_{0}$ and $A x_{0}$ are comparable.

Then $A$ has a fixed point $x^{*}$ which is unique in the comparable sense. Namely, if Ay* $y^{*}$ and $y^{*}$ and $x^{*}$ are comparable, then $y^{*}=x^{*}$. Moreover,

$$
d\left(x^{*}, x_{0}\right) \leq \frac{1}{1-k} N M_{\left(A x_{0}\right) x_{0}^{-1}} .
$$

Proof Define a sequence $\left\{x_{n}\right\}_{n \in \mathbb{N}} \subseteq E$ by the formula $x_{n}=A^{n} x_{0}, n \in \mathbb{N}$. The proof can be divided into three steps.

Step $I .\left\{x_{n}\right\}$ is a Cauchy sequence.

Now, since $x_{0}$ and $A x_{0}=x_{1}$ are comparable, and $A$ satisfies the $k$-ordered contractive condition, we obtain $x_{1}$ and $x_{2}, x_{2}$ and $x_{3}, \ldots, x_{n}$ and $x_{n+1}$ are comparable. Notice

$$
\vee\left(x_{n} x_{n+1}^{-1}, x_{n+1} x_{n}^{-1}\right) \lesssim \vee\left(x_{n-1} x_{n}^{-1}, x_{n} x_{n-1}^{-1}\right)^{k}
$$

Inductively the following holds:

$$
\vee\left(x_{1} x_{2}^{-1}, x_{2} x_{1}^{-1}\right) \lesssim \vee\left(x_{0} x_{1}^{-1}, x_{1} x_{0}^{-1}\right)^{k}
$$

By the above for all $n \in \mathbb{N}$ and Lemma 1.1(1), we obtain

$$
e \lesssim \vee\left(x_{n+1} x_{n}^{-1}, x_{n} x_{n+1}^{-1}\right) \lesssim \vee\left(x_{n} x_{n-1}^{-1}, x_{n-1} x_{n}^{-1}\right)^{k} \lesssim \cdots \lesssim \vee\left(x_{1} x_{0}^{-1}, x_{0} x_{1}^{-1}\right)^{k^{n}}
$$

From the above it is easy to conclude that

$$
d\left(\vee\left(x_{n+1} x_{n}^{-1}, x_{n} x_{n+1}^{-1}\right), e\right) \leq N d\left(\vee\left(x_{1} x_{0}^{-1}, x_{0} x_{1}^{-1}\right)^{k^{n}}, e\right),
$$


which together with Definition 1.1 yields

$$
d\left(\vee\left(x_{n+1} x_{n}^{-1}, x_{n} x_{n+1}^{-1}\right), e\right) \leq N M_{x_{1} x_{0}^{-1}} k^{n} .
$$

Finally, by Lemma 1.1(3), this implies

$$
d\left(x_{n}, x_{n+1}\right) \leq N M_{x_{1} x_{0}^{-1}} k^{n} \rightarrow 0,
$$

which shows $\left\{x_{n}\right\}$ is a Cauchy sequence. The completeness of $E$ implies that there exists $x^{*} \in E$ such that $\lim _{n \rightarrow \infty} x_{n}=x^{*}$.

Step II. $x^{*}$ is a fixed point of $A$.

Suppose that $\lim _{n \rightarrow \infty} x_{n}=x^{*}$, which together with $A$ is continuous, we get

$$
A x^{*}=A \lim _{n \rightarrow \infty} x_{n}=\lim _{n \rightarrow \infty} A x_{n}=x^{*},
$$

which shows $x^{*}$ is a fixed point of $A$.

Step III. The uniqueness of the fixed point of $A$ in the comparable sense.

Let us consider $A y^{*}=y^{*}$, and let $x^{*}$ and $y^{*}$ be comparable. Without loss of generality, set $y^{*} \lesssim x^{*}$, which shows $x^{*} y^{*-1} \in P$. By the condition (1),

$$
\vee\left(A y^{*}\left(A x^{*}\right)^{-1}, A x^{*}\left(A y^{*}\right)^{-1}\right) \lesssim \vee\left(y^{*} x^{*-1}, x^{*} y^{*-1}\right)^{k}
$$

That is

$$
\vee\left(y^{*} x^{*-1}, x^{*} y^{*-1}\right) \lesssim \vee\left(y^{*} x^{*-1}, x^{*} y^{*-1}\right)^{k}
$$

In addition, we know $\vee\left(y^{*} x^{*-1}, x^{*} y^{*-1}\right)=x^{*} y^{*-1}$. Then $x^{*} y^{*-1} \lesssim\left(x^{*} y^{*-1}\right)^{k}$, which implies $\left(x^{*} y^{*-1}\right)^{k-1} \in P$. On the other hand, we have $\left(x^{*} y^{*-1}\right)^{1-k} \in P$. Now, from the definition of a cone, we have $x^{*} y^{*-1}=e$, and then $y^{*}=x^{*}$.

Furthermore,

$$
d\left(x^{*}, x_{0}\right) \leq \sum_{n=0}^{\infty} d\left(x_{n}, x_{n+1}\right) \leq \sum_{n=0}^{\infty} N M_{\left(A x_{0}\right) x_{0}^{-1}} k^{n}=\frac{1}{1-k} N M_{\left(A x_{0}\right) x_{0}^{-1}}
$$

Remark 2.1 Assume in addition that $E$ satisfies the condition:

(3) For $u, v \in E$, if they are not comparable, then there exists $w \in E$ such that $u$ and $w, v$ and $w$ are comparable, respectively.

Then $A$ has a unique fixed point. Note that condition (3) is always valid if $E$ is a lattice. It suffices to show the uniqueness of the fixed point of $A$. Suppose that $x^{*}, y^{*}$ are fixed points of $A$. Claim that $x^{*}$ and $y^{*}$ are comparable. If not, there exists $z \in E$ such that $x^{*}$ and $z, y^{*}$ and $z$ are comparable, respectively. Since $A$ satisfies the $k$-ordered contractive condition, then $A^{n+1} x^{*}$ and $A^{n+1} z, A^{n+1} y^{*}$ and $A^{n+1} z$ are comparable for any $n \in \mathbb{N}$, respectively. Also,

$$
e \lesssim \vee\left(A^{n+1} x^{*}\left(A^{n+1} z\right)^{-1}, A^{n+1} z\left(A^{n+1} x^{*}\right)^{-1}\right) \lesssim \vee\left(x^{*} z^{-1}, z\left(x^{*}\right)^{-1}\right)^{k^{n}}
$$


and

$$
e \lesssim \vee\left(A^{n+1} y^{*}\left(A^{n+1} z\right)^{-1}, A^{n+1} z\left(A^{n+1} y^{*}\right)^{-1}\right) \lesssim \vee\left(y^{*} z^{-1}, z\left(y^{*}\right)^{-1}\right)^{k^{n}}
$$

Then

$$
\begin{aligned}
d\left(A^{n+1} x^{*}, A^{n+1} z\right) & =d\left(\vee\left(A^{n+1} x^{*}\left(A^{n+1} z\right)^{-1}, A^{n+1} z\left(A^{n+1} x^{*}\right)^{-1}\right), e\right) \\
& \leq N d\left(\vee\left(x^{*} z^{-1}, z\left(x^{*}\right)^{-1}\right)^{k^{n}}, e\right) \\
& \leq N k^{n} M_{x^{*} z^{-1}}
\end{aligned}
$$

and similarly

$$
d\left(A^{n+1} y^{*}, A^{n+1} z\right) \leq N k^{n} M_{y^{*} z^{-1}}
$$

The triangular inequality tells us that

$$
\begin{aligned}
d\left(x^{*}, y^{*}\right) & =d\left(A^{n+1} x^{*}, A^{n+1} y^{*}\right) \\
& \leq d\left(A^{n+1} x^{*}, A^{n+1} z\right)+d\left(A^{n+1} y^{*}, A^{n+1} z\right) \\
& \leq N k^{n}\left(M_{x^{*} z^{-1}}+M_{y^{*} z^{-1}}\right) \rightarrow 0,
\end{aligned}
$$

as $n \rightarrow \infty$, which implies $x^{*}=y^{*}$. This is a contradiction. Therefore, $x^{*}$ and $y^{*}$ are comparable. By Theorem 2.1, $x^{*}=y^{*}$.

Corollary 2.1 Let E be a uniformly bounded noncommutative Banach space, $P$ a normal cone with the normal constant $N$. For $c \in \mathbb{R}^{+}, x_{0} \in E$, set $B\left(x_{0}, c\right)=\left\{x \in E: d\left(x, x_{0}\right) \leq c\right\}$. Suppose that a continuous mapping $A: E \rightarrow$ E satisfies the $k$-ordered contractive condition and $d\left(A x_{0}, x_{0}\right) \leq c-k N M$. Also, $x_{0}$ and $x \in B\left(x_{0}, c\right)$ are comparable. Then there exists a unique fixed point in $B\left(x_{0}, c\right)$ in the comparable sense.

Proof It suffices to show that $A x \in B\left(x_{0}, c\right)$ for any $x \in B\left(x_{0}, c\right)$.

For any $x \in B\left(x_{0}, c\right)$, the triangular inequality gives

$$
d\left(x_{0}, A x\right) \leq d\left(x_{0}, A x_{0}\right)+d\left(A x_{0}, A x\right) .
$$

By Lemma 1.1(3),

$$
d\left(A x_{0}, A x\right)=d\left(\vee\left(A x_{0}(A x)^{-1}, A x\left(A x_{0}\right)^{-1}\right), e\right) .
$$

Since $A$ satisfies the $k$-ordered contractive condition, we have

$$
\vee\left(A x_{0}(A x)^{-1}, A x\left(A x_{0}\right)^{-1}\right) \lesssim \vee\left(x_{0} x^{-1}, x x_{0}^{-1}\right)^{k}
$$

and then

$$
d\left(\vee\left(A x_{0}(A x)^{-1}, A x\left(A x_{0}\right)^{-1}\right), e\right) \leq N d\left(\vee\left(x_{0} x^{-1}, x x_{0}^{-1}\right)^{k}, e\right) \leq k N M
$$


Hence we get

$$
d\left(x_{0}, A x\right) \leq d\left(x_{0}, A x_{0}\right)+d\left(A x_{0}, A x\right) \leq c-k N M+k N M=c,
$$

from which one deduces that

$$
A x \in B\left(x_{0}, c\right) .
$$

Corollary 2.2 Let $A: E \rightarrow E$ be a continuous mapping and suppose that the following two assertions hold:

(1) there exist $k \in(0,1)$ and $n_{0} \in \mathbb{N}$ such that for all $u, v \in E$, if $u$ and $v$ are comparable, then $A u$ and $A v$ are comparable and furthermore

$$
\vee\left(A^{n_{0}} v\left(A^{n_{0}} u\right)^{-1}, A^{n_{0}} u\left(A^{n_{0}} v\right)^{-1}\right) \lesssim \vee\left(v u^{-1}, u v^{-1}\right)^{k}
$$

(2) there exists $x_{0} \in E$ such that $x_{0}$ and $A x_{0}$ are comparable.

Then $A$ admits a unique fixed point $x^{*}$ in the comparable sense.

Proof By Theorem 2.1, we know $A^{n_{0}}$ has a unique fixed point $x^{*}$ in the comparable sense. Notice that

$$
A^{n_{0}}\left(A x^{*}\right)=A\left(A^{n_{0}} x^{*}\right)=A x^{*}
$$

which means $A x^{*}$ is also a fixed point of $A^{n_{0}}$. Again, since $x_{0}$ and $A x_{0}$ are comparable, then $x^{*}$ and $A x^{*}$ are comparable, which implies $A x^{*}=x^{*}$. Since the fixed point of $A$ is also the fixed point of $A^{n_{0}}$, the fixed point of $A$ is unique in the comparable sense.

Theorem 2.2 Let $A: E \rightarrow$ E be a mapping and suppose that the following two assertions hold:

(1) there exists a constant $k \in\left(0, \frac{1}{2}\right)$ such that for all $\beta \in[0,1]$, if $u$ and $v$ are comparable, then $A u$ and $A v, A u$ and $u, A v$ and $v$ are comparable, and furthermore

$$
\vee\left(A v(A u)^{-1}, A u(A v)^{-1}\right)^{\beta} \lesssim \vee\left(A u u^{-1}, u(A u)^{-1}\right)^{k \beta} \vee\left(A v v^{-1}, v(A v)^{-1}\right)^{k \beta}
$$

(2) A is continuous.

Then $A$ admits a unique fixed point $x^{*}$ in the comparable sense, and for each $x_{0} \in E$, $\lim _{n \rightarrow \infty} A^{n} x_{0}=x^{*}$. Moreover,

$$
d\left(x^{*}, x_{0}\right) \leq \frac{1-k}{1-2 k} N M_{\left(A x_{0}\right) x_{0}^{-1}}
$$

Proof By the reflexivity of the partial ordering ' $\lesssim$ in $E, x_{0} \lesssim x_{0}$. And since $A$ satisfies the condition (1), then $x_{0}$ and $A x_{0}, A x_{0}$ and $A^{2} x_{0}, \ldots, A^{n} x_{0}$ and $A^{n+1} x_{0}$ are comparable. Put $x_{n}=A^{n} x_{0}$. Then for each integer $n \geq 1$, from the condition (1), we get

$$
\begin{aligned}
\vee\left(x_{n} x_{n+1}^{-1}, x_{n+1} x_{n}^{-1}\right)^{\beta} & =\vee\left(A x_{n-1}\left(A x_{n}\right)^{-1}, A x_{n}\left(A x_{n-1}\right)^{-1}\right)^{\beta} \\
& \lesssim \vee\left(A x_{n-1} x_{n-1}^{-1}, x_{n-1}\left(A x_{n-1}\right)^{-1}\right)^{k \beta} \vee\left(A x_{n} x_{n}^{-1}, x_{n}\left(A x_{n}\right)^{-1}\right)^{k \beta} \\
& =\vee\left(x_{n} x_{n-1}^{-1}, x_{n-1} x_{n}^{-1}\right)^{k \beta} \vee\left(x_{n+1} x_{n}^{-1}, x_{n} x_{n+1}^{-1}\right)^{k \beta} .
\end{aligned}
$$


By the definition of partial ordering in $E$ with respect to $P$, one has

$$
\vee\left(x_{n} x_{n-1}^{-1}, x_{n-1} x_{n}^{-1}\right)^{k \beta} \vee\left(x_{n+1} x_{n}^{-1}, x_{n} x_{n+1}^{-1}\right)^{-(1-k) \beta} \in P .
$$

That is

$$
\vee\left(x_{n+1} x_{n}^{-1}, x_{n} x_{n+1}^{-1}\right)^{1-k} \lesssim \vee\left(x_{n} x_{n-1}^{-1}, x_{n-1} x_{n}^{-1}\right)^{k}
$$

From Lemma 1.1(1), one can obtain

$$
\vee\left(x_{n+1} x_{n}^{-1}, x_{n} x_{n+1}^{-1}\right) \lesssim \vee\left(x_{n} x_{n-1}^{-1}, x_{n-1} x_{n}^{-1}\right)^{\frac{k}{1-k}}
$$

As in the proof of Theorem 2.1, $\left\{x_{n}\right\}$ is a Cauchy sequence and there exists $x^{*} \in E$ such that $\lim _{n \rightarrow \infty} x_{n}=x^{*}$. Also, $x^{*}$ is a fixed point of $A$.

It remains to be shown that $x^{*}$ is a unique fixed point of $A$.

Suppose that there exists $y^{*} \in E, y^{*} \lesssim x^{*}$, such that $A y^{*}=y^{*}$. Due to the condition (1), we have

$$
\vee\left(A y^{*}\left(A x^{*}\right)^{-1}, A x^{*}\left(A y^{*}\right)^{-1}\right) \lesssim \vee\left(A y^{*} y^{*-1}, y^{*}\left(A y^{*}\right)^{-1}\right)^{k} \vee\left(A x^{*} x^{*-1}, x^{*}\left(A x^{*}\right)^{-1}\right)^{k}
$$

That is

$$
\vee\left(y^{*} x^{*-1}, x^{*} y^{*-1}\right) \lesssim \vee\left(y^{*} y^{*-1}, y^{*} y^{*-1}\right)^{k} \vee\left(x^{*} x^{*-1}, x^{*} x^{*-1}\right)^{k}=e
$$

Since $\vee\left(y^{*} x^{*-1}, x^{*} y^{*-1}\right)=x^{*} y^{*-1}$. Then $x^{*} y^{*-1} \lesssim e$, which implies $\left(x^{*} y^{*-1}\right)^{-1} \in P$. Now, applying Definition 1.2, we obtain the desired result.

Similar to the proof of Theorem 2.1, one can verify

$$
d\left(x^{*}, x_{0}\right) \leq \frac{1-k}{1-2 k} N M_{\left(A x_{0}\right) x_{0}^{-1}} .
$$

Let $u_{0}, v_{0} \in E$, and $u_{0} \lesssim v_{0}$. By $\left[u_{0}, v_{0}\right]$ from now on we denote the order interval $\{u \in E$ : $\left.u_{0} \lesssim u \lesssim v_{0}\right\}$. Finally, we consider the fixed-point theorem in the order interval.

Theorem 2.3 Let $\left[u_{0}, v_{0}\right]$ be the order interval in E. If $A:\left[u_{0}, v_{0}\right] \rightarrow\left[u_{0}, v_{0}\right]$ satisfies the conditions in Theorem 2.2, then $A$ has a unique fixed point in the comparable sense.

Proof Consider sequences $\left\{u_{n}\right\}$ and $\left\{v_{n}\right\}$ defined by $u_{n}=A^{n} u_{0}$ and $v_{n}=A^{n} v_{0}$, respectively. Then $\left\{u_{n}\right\},\left\{v_{n}\right\} \subseteq\left[u_{0}, v_{0}\right]$. Since $A$ satisfies Theorem 2.2(1), then for any $n \in \mathbb{N}, u_{n}$ and $v_{n}$ are comparable, and

$$
e \lesssim \vee\left(u_{n} v_{n}{ }^{-1}, v_{n} u_{n}{ }^{-1}\right) \lesssim \vee\left(u_{0} v_{0}{ }^{-1}, v_{0} u_{0}{ }^{-1}\right)^{\left(\frac{k}{1-k}\right)^{n}} .
$$

Notice that $P$ is a normal cone,

$$
d\left(u_{n}, v_{n}\right) \leq N M_{u_{0} v_{0}^{-1}}\left(\frac{k}{1-k}\right)^{n}
$$


Again $u_{0} \lesssim u_{1}$, hence for all $n \in \mathbb{N}, u_{n}$ and $u_{n+1}$ are comparable. Then

$$
e \lesssim \vee\left(u_{n} u_{n+1}{ }^{-1}, u_{n+1} u_{n}^{-1}\right) \lesssim \vee\left(u_{0} u_{1}^{-1}, u_{1} u_{0}^{-1}\right)^{\left(\frac{k}{1-k}\right)^{n}}
$$

from which one deduces

$$
d\left(u_{n}, u_{n+1}\right) \leq N M_{u_{1} u_{0}-1}\left(\frac{k}{1-k}\right)^{n}
$$

Hence, we conclude that $\left\{u_{n}\right\}$ is a Cauchy sequence with a limit point $u^{*} \in\left[u_{0}, v_{0}\right]$ for any $0<k<\frac{1}{2}$.

Similarly, $\left\{v_{n}\right\}$ is a Cauchy sequence with a limit point $v^{*} \in\left[u_{0}, v_{0}\right]$ for any $0<k<\frac{1}{2}$.

Now, as

$$
d\left(u^{*}, v^{*}\right)=\lim _{n \rightarrow \infty} d\left(u_{n}, v_{n}\right) \leq \lim _{n \rightarrow \infty} N M_{u_{0} v_{0}^{-1}}\left(\frac{k}{1-k}\right)^{n}=0
$$

then $u^{*}=v^{*}$.

The rest of the proof is analogue to that in Theorem 2.2.

If one checks the proof of Theorem 2.3 , then one can easily obtain the following result.

Corollary 2.3 Let $\left[u_{0}, v_{0}\right]$ be the order interval in E. If the continuous mapping A : $\left[u_{0}, v_{0}\right] \rightarrow\left[u_{0}, v_{0}\right]$ satisfies the $k$-ordered contractive condition, then $A$ admits a unique fixed point in the comparable sense.

\section{Examples}

We give some examples to illustrate the main result of this paper in the following.

Example 3.1 Consider the space $\left(\mathbb{R}^{n}, d\right)$ given in Example 1.2. Let $P=\left\{x=\left(x_{1}, x_{2}, \ldots, x_{n}\right) \in\right.$ $\left.\mathbb{R}^{n}: x_{i} \geq 0,1 \leq i \leq n\right\}$ be a normal cone. The partial ordering in $E$ with respect to $P$ is given by

$$
x \lesssim y \quad \Longleftrightarrow \quad x_{i} \leq y_{i}, \quad 1 \leq i \leq n
$$

then $E$ is a lattice. It is known that the operators on $\mathbb{R}^{n}$ are in one-to-one correspondence with the $n \times n$ matrices. Consider a matrix

$$
A=\left(\begin{array}{ccccc}
a_{1} & 0 & 0 & \cdots & 0 \\
0 & a_{2} & 0 & \cdots & 0 \\
\cdot & \cdot & \cdot & \cdots & \cdot \\
\cdot & \cdot & \cdot & \cdots & \cdot \\
0 & 0 & 0 & \cdots & a_{n}
\end{array}\right),
$$

where $0<a_{i}<1, i=1,2, \ldots, n$. Choose $k \in \mathbb{R}$ such that $\max _{1 \leq i \leq n}\left\{a_{i}\right\}<k<1$. Clearly, $A x$ and $A y$ are comparable if $x$ and $y$ are comparable. Moreover, if $x \lesssim y$, then $A y-A x \lesssim k(y-x)$. By Theorem 2.1 and Remark 2.1, we know that $A$ has a unique fixed point. 
Example 3.2 Consider the integral equation of Fredholm type

$$
x(t)=\int_{0}^{1} K(t, s, x(s)) d s+g(t), \quad t \in[0,1] .
$$

Assume that

(1) $K:[0,1] \times[0,1] \times \mathbb{R} \rightarrow \mathbb{R}$ and $g:[0,1] \rightarrow \mathbb{R}$ are continuous;

(2) $K(t, s, \cdot): \mathbb{R} \rightarrow \mathbb{R}$ is monotonous for $t, s \in \mathbb{R}$;

(3) there exist a continuous function $\varphi:[0,1] \times[0,1] \rightarrow \mathbb{R}$ and $k \in(0,1)$ such that

$$
|K(t, s, u)-K(t, s, v)| \leq k|\varphi(t, s)(u-v)|,
$$

for $t, s \in[0,1], u, v \in \mathbb{R}$.

(4) $\sup _{t \in[0,1]} \int_{0}^{1}|\varphi(t, s)| d s \leq 1$;

(5) there exists $x_{0} \in C[0,1]$ such that for any $t \in[0,1], x_{0}(t) \leq \int_{0}^{1} K\left(t, s, x_{0}(s)\right) d s+g(t)$ or $x_{0}(t) \geq \int_{0}^{1} K\left(t, s, x_{0}(s)\right) d s+g(t)$.

Then the integral equation has a unique solution $x^{*}$ in $C[0,1]$.

In fact, let $E=C[0,1]$ with the metric induced by the supremum norm, i.e.,

$$
d(x, y)=\|x-y\|=\max \{|x(t)-y(t)|: t \in[0,1]\}
$$

for $x, y \in C[0,1]$, and $P=\{x \in C[0,1]: x(t) \geq 0$ for any $t \in[0,1]\}$ be a normal cone in $C[0,1]$. The partial ordering in $E$ induced by $P$ is given as follows:

$$
x \lesssim y \quad \Longleftrightarrow \quad x(t) \leq y(t) \quad \text { for any } t \in[0,1] .
$$

Define $A: C[0,1] \rightarrow C[0,1]$ by

$$
A(x(t))=\int_{0}^{1} K(t, s, x(s)) d s+g(t), \quad t \in[0,1] .
$$

From the condition (2), $A$ is monotonous. The monotonicity condition implies that $A x$ and $A y$ are comparable if $x$ and $y$ are comparable. Observe that for $x, y \in E$, if $x$ and $y$ are comparable, then

$$
\begin{aligned}
|A(y(t))-A(x(t))| & \leq \int_{0}^{1}|K(t, s, y(s))-K(t, s, x(s))| d s \\
& \leq k \int_{0}^{1}|\varphi(t, s)(y(s)-x(s))| d s \\
& \leq k \int_{0}^{1}|\varphi(t, s)| d s\|y-x\| \\
& \leq k\|y-x\|,
\end{aligned}
$$

for $t \in[0,1]$, which implies that $\|A y-A x\| \leq k\|y-x\|$. Thus, $A$ is continuous. Again, $E$ is a lattice, by Theorem 2.1 and Remark 2.1, the integral equation has a unique solution $x^{*}$ in $C[0,1]$. 
Observe that the functions satisfying the conditions in Example 3.2 do exist. For example,

$$
\begin{aligned}
& K(t, s, u)=k t s u, \quad \text { where } k \in(0,1) \\
& g(t) \geq 0 \quad \text { or } \quad g(t) \leq 0 \quad \text { is continuous, } \\
& \varphi(t, s)=t s \\
& x_{0}(t)=0
\end{aligned}
$$

for $t, s \in[0,1]$ and $u \in \mathbb{R}$.

Notice that the examples given above are in linear spaces. As to the noncommutative case, it is under consideration now.

\section{Competing interests}

The authors declare that they have no competing interests.

\section{Authors' contributions}

The authors contributed equally and significantly in writing this article. All authors read and approved the final manuscript.

\section{Acknowledgements}

The authors would like to thank the referees for their many valuable suggestions that have greatly contributed to improve the quality of this paper. This work is supported financially by the NSFC $(10971011,11371222)$.

Received: 10 September 2013 Accepted: 21 January 2014 Published: 07 Feb 2014

\section{References}

1. Banach, S: Sur les opérations dans les ensembles abstraits et leur application aux équations intégrales. Fundam. Math. 3, 133-181 (1922)

2. Abbas, M, Rhoades, BE, Nazir, T: Common fixed points for four maps in cone metric spaces. Appl. Math. Comput. 216, 80-86 (2010)

3. Agarwal, RP, El-Gebeily, MA, O’Regan, D: Generalized contractions in partially ordered metric spaces. Appl. Anal. 87(1), 109-116 (2008)

4. Altun, I, Damnjanović, B, Djorić, D: Fixed point and common fixed point theorems on ordered cone metric spaces. Appl. Math. Lett. 23, 310-316 (2010)

5. Amini-Harandi, A, Emami, H: A fixed point theorem for contraction type maps in partially ordered metric spaces and application to ordinary differential equations. Nonlinear Anal. 72(5), 2238-2242 (2010)

6. Caballero, J, Harjani, J, Sadarangani, K: Contractive-like mapping principles in ordered metric spaces and application to ordinary differential equations. Fixed Point Theory Appl. 2010, Article ID 916064 (2010)

7. Cho, YJ, Saadati, R, Wang, SH: Common fixed point theorems on generalized distance in ordered cone metric spaces. Comput. Math. Appl. 61, 1254-1260 (2011)

8. Dhage, BC, O'Regan, D, Agrawal, RP: Common fixed point theorems for a pair of countably condensing mapping in ordered Banach spaces. J. Appl. Math. Stoch. Anal. 62(2), 344-348 (1977)

9. Huang, LG, Zhang, X: Cone metric spaces and fixed point theorems of contractive mappings. J. Math. Anal. Appl. 33(2), 1468-1476 (2007)

10. Kadelburg, Z, Pavlović, M, Radenović, S: Common fixed point theorems for ordered contractions and quasicontractions in ordered cone metric spaces. Comput. Math. Appl. 59, 3148-3159 (2010)

11. Klim, D, Wardowski, D: Dynamic processes and fixed points of set-valued nonlinear contractions in cone metric spaces. Nonlinear Anal. 71, 5170-5175 (2009)

12. O'Regan, D, Petruşel, A: Fixed point theorems for generalized contractions in ordered metric spaces. J. Math. Anal. Appl. 341(2), 1241-1252 (2008)

13. Ran, ACM, Reurings, MCB: A fixed point theorem in partially ordered sets and some applications to matrix equations. Proc. Am. Math. Soc. 132, 1435-1443 (2004)

14. Wang, JG: Fixed points of increasing operators in ordered Banach spaces. Acta Math. Sin. Chin. Ser. 43, 43-48 (2000)

10.1186/1687-1812-2014-30

Cite this article as: Xin and Jiang: Fixed-point theorems for mappings satisfying the ordered contractive condition on noncommutative spaces. Fixed Point Theory and Applications 2014, 2014:30 